\title{
Does timing of completion radical cholecystectomy determine the survival outcome in incidental carcinoma gallbladder: A single center retrospective analysis
}

\author{
Rahul', Kulbhushan HALDENIYA', Ashish SINGH', Prabhakar MISHRA², \\ Rajneesh Kumar SINGH', Anu BEHARI', Vinay Kumar KAPOOR', Rajan SAXENA**1 \\ 'Department of Surgical Gastroenterology, Sanjay Gandhi Post Graduate Institute of Medical Sciences, Lucknow, India \\ ${ }^{2}$ Department of Biostatistics and Health Informatics, Sanjay Gandhi Post Graduate Institute of Medical Sciences, Lucknow, India
}

Introduction: Incidental discovery of gallbladder cancer (GBC) on postoperative histopathology or intra-operative suspicion is becoming increasingly frequent, since laparoscopic cholecystectomy became the standard of care for gall stone disease. Incidental GBC (IGBC) portends a better survival than primarily detected GBC. Various factors affect the outcome of re-resection with timing of re-intervention an important determinant of survival.

Methods: All patients of IGBC who underwent curative resection from January 2009 to December 2018 were considered for analysis. Details of demographic profile, index surgery, primary histopathology, operative findings on re-resection, final histopathology and follow-up data were retrieved from the prospectively maintained database. Patients were evaluated in three groups based on the interval between index cholecystectomy and re-intervention (early [<4weeks], intermediate [4-12 weeks], and late [>12 weeks]) using appropriate statistical tests.

Results: Forty eight patients underwent re-resection with curative intent. Median age of presentation was 55 years. Mean and median follow-up was 51.6 and 40.6 months respectively (range, 1.2-130.6 months). The overall survival and disease free survival among the three groups was the best in 'early' group (104 \& 102 months) as compared to the 'Intermediate' (84 \& 83 months) and 'late' groups (75 $\& 73$ months), though the difference was not statistically significant $(p=0.588$ and $p=0.581)$. On Multivariate analysis, poor differentiation was the only independent factor affecting survival. Other attributes which were associated with poor outcome, but could not reach statistical significance were node metastasis, delay in re-resection, residual tumor, need for CBD excision and lymphovascular invasion.

Conclusions: Grade of tumor is the most important determinant of survival in IGBC. Early surgery, preferably within 4 weeks possibly entails better survival. 